hep-th/0205288

SU-ITP-02/18

SLAC-PUB-9213

\title{
On the instability of $3 \mathrm{~d}$ null singularities
}

\author{
Albion Lawrence \\ SLAC Theory Group, MS 81, 2575 Sand Hill Road, Menlo Park, CA 94025, and \\ Department of Physics, Stanford University, Stanford, CA 94305.
}

String propagation on a three-dimensional Lorentzian string orbifold with a null singularity has been studied by Horowitz and Steif, and more recently by Liu, Moore and Seiberg. We analyze the target space as a classical gravitational background. The singularity becomes spacelike when an arbitrarily small amount of matter is thrown at the singularity. This can be seen directly by constructing a Vaidya-type solution, or by studying the null singularity as a limit of the $M=0, J=0$ BTZ black hole metric.

May 2002 


\section{Introduction}

String theory has had great success resolving singularities in classical supergravity which occur at a point in space. But time-dependent backgrounds are poorly understood, and the fate of dynamical or spacelike singularities is unknown. Since spacelike singularities arise from reasonable initial conditions in classical general relativity [1], they are an important feature of gravitational dynamics. Furthermore, there are good reasons to believe that the physics of spacelike gravitational singularities is qualitatively different than that of timelike singularities (c.f. [2]).

A handful of exactly solvable conformal field theories corresponding to strings propagating in time dependent backgrounds with spacelike or null singularities have been con-

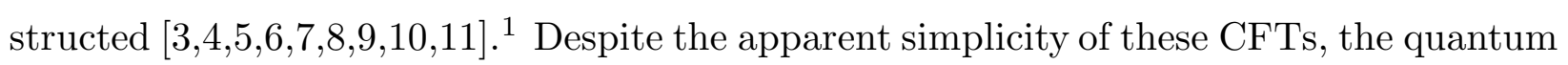
dynamics of strings remains unclear. The target spaces of many of these CFTs have closed timelike curves [9,8, 11], and string propagation is singular at many of these singularities [15,4.

It is not even clear whether conformal field theory is the correct tool for discussing such singularities. The dynamical singularities demanded by the singularity theorems signal a disease in the evolution of classical gravitational fields. The classical limit of string theory - the limit of large vevs of quantum fields - is not yet understood, particularly in the closed string sector. There are no real principles for continuing the spacetime beyond the singularity, nor is it obvious that continuing past the singularity is desirable.

A related issue is that quantum string production and the resulting backreaction could be large. For example, in the spacetimes described by [6,9], demanding that spacetime quantum fields are invariant under the orbifold group at the orbifold fixed points implies large particle production at the singularity [16]. Quantum string production in timedependent backgrounds is still a young subject (see [17 for the most complete discussion to date), 2 but preliminary results suggest that near singularities, the backreaction from string production may be even more drastic than that from field theory [18].

The purpose of this work is to argue that in the spacetime background described in [4, 10], the dynamics of a probe and the classical formation of spacelike singularities are

1 Conformal field theories describing string propagation in time-dependent bacgkrounds with timelike or no singularities have been constructed in [12, 13, 14].

2 See also [14 for a spacetime field-theoretic calculation of string production in a timedependent background. 
not separable issues. On the one hand, the target space studied is an explicit orbifold of string theory, so it is under control as a conformal field theory. It has a null singularity and no closed timelike curves, and the presence of a null Killing vector insures that there is no particle production [19]. The system seems to be an ideal laboratory for studying time-dependent backgrounds using conformal field theory techniques.

On the other hand, the causal structure of the singularity is unstable. Any stressenergy satisfying the positive or null energy condition, at arbitrarily small but finite energy in three dimensions, will replace the singularity with a spacelike singularity, of the kind described in $4,20,21$.

We will show this first by studying null stress energy impinging on the singularity of [4, 10], in the classical gravitational limit in three dimensions. We will then argue that this behavior is reasonable and generic by studying the solution as a particular limit of the BTZ black hole metric. We will conclude with a brief discussion of string amplitudes in this background.

\section{Collapsing null matter}

The metric of the orbifold in 4,10 , can be written after a suitable change of coordinates as: $: 3$

$$
d s^{2}=-2 d y^{+} d y^{-}+\left(y^{+}\right)^{2} d y^{2}
$$

where $y \sim y+2 \pi$ is a periodic variable. For $y^{+}<0$, this spacetime has the structure of a $2 \mathrm{~d}$ Lorentzian cone times a lightlike line. It is unclear whether the $y^{+}>0$ region should be kept; we will not address that issue here.

To study the effects of matter impinging on the singularity, we will send in a pulse of null stress energy, which is circularly symmetric - that is, translationally invariant along $y$. We take the energy-momentum tensor to be:

$$
T_{--}=\alpha \frac{g\left(y^{-}\right)}{8 \pi G_{N}^{(3)}\left(-y^{+}\right)},
$$

3 These are the "y-coordinates" in [10]. The singular submanifold at $y^{+}=0$ has a different structure than the singularity in the original coordinates, since the coordinate transformation is bad there. This will not effect the calculation in this paper, which describes the spacetime at any point off of the singular locus. Of course it may be important for the resolution of the singularity. 
which describes null matter striking the singularity. The dependence on $y^{+}$is required for $T_{\mu \nu}$ to be conserved. Here $G_{N}^{(3)}$ is the 3-dimensional Newton's constant. We assume that $g\left(y^{+}\right)$is positive definite, and that

$$
\int_{-\infty}^{\infty} d y^{-} g\left(y^{-}\right)=1
$$

If the circular shell is approaching from $y^{+}<0$, the energy of the incoming shell is positive if $\alpha>0$.

Taking the ansätz for the metric:

$$
d s^{2}=f\left(y^{-}\right)\left(d y^{-}\right)^{2}-2 d y^{-} d y^{+}+\left(y^{+}\right)^{2} d y^{2},
$$

the solution to Einstein's equation is:

$$
f\left(y^{-}\right)=-\alpha \int_{-\infty}^{y^{-}} d x g(x) .
$$

Assume $g$ has compact support in the interval $y^{-} \in[0, a]$. For $y^{-} \leq 0$, the metric is that of the null singularity in [10]. For $y \geq a$ the metric is:

$$
d s^{2}=\alpha\left(d y^{-}\right)^{2}-2 d y^{+} d y^{-}+\left(y^{+}\right)^{2} d y^{2} .
$$

If $\alpha>0$, so that the energy of the incoming shell is positive, then $y^{+}=0$ has become a spacelike singular locus. $y$ describes a circle whose circumference shrinks with the time coordinate $y^{+}$. A further coordinate transformation makes the structure clear:

$$
\begin{aligned}
u & =\frac{1}{\alpha}(\tau+\sigma) \\
v & =\tau \\
\phi & =\sqrt{\alpha} y .
\end{aligned}
$$

The metric is:

$$
d s^{2}=\frac{1}{\alpha}\left(-d \tau^{2}+d \sigma^{2}+\tau^{2} d \phi^{2}\right)
$$

where $\psi \sim \phi+2 \pi \sqrt{\alpha}$. The spacelike singularity at $\tau=0$ is of the type discussed in [4, 20,21, 6].

This instability in the causal structure can be understood by studying the effects of collapsing massive dust in $2+1$ dimensions [22]. For a given velocity of the shell, there is a critical value $\mu_{c}$ of the mass of the shell, at which the metric outside the shell approaches that of the null singularity in [4, 10] as the shell shrinks to zero size. For any mass smaller than the critical value, the endpoint is a conical singularity (as expected for a point mass below the critical value $\mu_{c}$.) For a range of masses larger than the critical value, the endpoint of the collapse is the metric (2.6). 


\section{The BTZ black hole}

As noted in [7,10, the null singularity (2.1) is a particular limit of the $M=0, J=0$ BTZ black hole. This provides a nice picture of the results of the previous section.

The BTZ black hole with positive mass $M$ and zero angular momentum $J$ has the metric [23]:

$$
d s^{2}=-\left(\frac{r^{2}}{\ell^{2}}-\epsilon\right) d t^{2}+\left(\frac{r^{2}}{\ell^{2}}-\epsilon\right)^{-1} d r^{2}+r^{2} d \phi^{2} .
$$

For $\epsilon>0$, this is a black hole with mass

$$
M=\frac{\epsilon}{8 G_{N}^{(3)}},
$$

in anti-de Sitter space with cosmological constant

$$
\Lambda=-\frac{1}{\ell^{2}} .
$$

The horizon is at $r=\sqrt{\epsilon} \ell$, and the singularity is at $r=0$. As $\ell \rightarrow \infty$ the horizon recedes to infinity.

The metric (2.1) can be found by taking the limit $\epsilon \rightarrow 0$ while focusing on the region near the singularity. If $r \ll \sqrt{\epsilon} \ell$, the metric approaches

$$
-\frac{1}{\epsilon} d r^{2}+\epsilon d t^{2}+r^{2} d \phi^{2}
$$

In particular, if we let

$$
\begin{aligned}
& t=\frac{y^{+}}{\epsilon} \\
& r=\epsilon\left(y^{-}+t\right)=\epsilon y^{-}+y^{+},
\end{aligned}
$$

then (3.4) is clearly equivalent to (2.1). For fixed $y^{+}, y^{-}$, this scaling limit is consistent so long as $\ell$ is large enough. As $\ell \rightarrow \infty$ one recovers 3 d gravity without a cosmological constant.

The instability of the null singularity is thus a limiting case of the fact that throwing matter at an extreme, massless BTZ black hole with a null singularity creates a massive black hole with a spacelike singularity and an event horizon. Observers placed so that they are inside the horizon after the matter passes them, eventually see the spacetime described in (2.6). If $\ell$ is finite, a black hole solution with a horizon exists; observers which are far enough away will see the formation of a classically static black hole with an event horizon. 


\section{Conclusions}

Our discussion has been entirely three-dimensional, as would be natural if the remaining seven dimensions were compactified. If additional dimensions are not compactified and the incoming probe is localized in these dimensions, the solution will not be as simple. A simpler question is, whether Planckian physics generically becomes important to the physics of actual higher-dimensional probes near the singularity. scalar field in $d$ dimensions, one can follow the arguments in [4], by solving the equations of motion in the spacetime (2.1), as is done in [10], and computing the energy-momentum tensor for a given mode. As in the presence of the spacelike singularities studied in [4], a classical mode of a scalar field in the spacetime (2.1) has diverging energy near $y^{+}=0$. This "instability" is more drastic than the effect we have described in $\S 2$, where we have assumed an idealized energy-momentum tensor with a total energy which is finite even near the singularity.

Because the null singularity is marginal, classical probes with very small incoming three-dimensional energy will change the causal structure of the singularity. This change is potentially serious; for example, once the singularity becomes spacelike there can be large particle production near the singularity [16]. One might hope that a signal of this change appears in string perturbation theory, or at least as an interesting disease in the perturbation expansion. It would be interesting to study this further, along the lines of [10].

However, the interpretation of worldsheet computations is unclear once the spacelike singularity develops. If it is natural to continue through the singularity at $y^{+}=0$, as advocated by [8,11] and suggested by [10], then one may set up scattering data far from the singularity 11]; but it is not clear if this continuation is natural or unique.

Absent this continuation, there is no obvious S-matrix interpretation of worldsheet correlation functions due to the future singularity. One may try to keep a version of the S-matrix by keeping the cosmological constant $1 / \ell$ small but finite.5 The $\epsilon \rightarrow 0$ limit of (3.1) is an orbifold of anti-de Sitter space [23]. Correlators of vertex operators specified by their behavior at the boundary of AdS then provide an analog of S-matrix elements.

4 This point arose during a conversation with T. Banks, J. Polchinski, S. Shenker, E. Silverstein and L. Susskind; see also the conclusions in [10]. The calculation described in in the following sentences was inspired by comments by J. Polchinski.

5 I am grateful to S. Shenker for asking about and discussing this point. 
An incoming string will create an $M>0$ AdS black hole with the spacelike singularity hidden by a horizon from the asymptotic observer. The nontriviality of the order of limits is consistent with the principles of black hole complementarity [24].

\section{Acknowledgments}

I am grateful to A. Adams, M. Fabinger, S. Hellerman, V. Hubeny, S. Kachru, N. Kaloper, M. Kleban, J. McGreevy, J. Polchinski, S. Shenker, E. Silverstein and L. Susskind for discussions. Thanks in particular to J.M., J.P. and E.S. for comments on the draft. This work is supported by NSF grant PHY-9870115, by the Stanford Institute for Theoretical Physics, and by the DOE under contract DE-AC03-76SF00515. 


\section{References}

[1] R. Penrose, Phys. Rev. Lett. 14 (1965) 57; S. Hawking, Proc. Roy. Soc. A300 (1967) 182; S. Hawking and R. Penrose, Proc. Roy. Soc. A314 (1970) 589.

[2] G.T. Horowitz and R.C. Myers, "The value of singularities," Gen. Rel. Grav. 27 (1995) 915; gr-qc/9503062.

[3] E. Witten, "On string theory and black holes," Phys. Rev. D44 (1991) 314.

[4] G.T. Horowitz and A.R. Steif, "Singular string solutions with nonsingular initial data," Phys. Lett. B258 (1991) 91.

[5] C.R. Nappi and E. Witten, "A closed, expanding universe in string theory," Phys. Lett. B293 (1992) 309, hep-th/9206078.

[6] N.A. Nekrasov, "Milne universe, tachyons and quantum group," hep-th/0203112.

[7] J. Simon, "The geometry of null rotation identifications," hep-th/0203201.

[8] S. Elitzur, A. Giveon, D. Kutasov and E. Rabinovici, "From big bang to big crunch and beyond," hep-th/0204189.

[9] V. Balasubramanian, S.F. Hassan, E. Keski-Vakkuri and A. Naqvi, "A space-time orbifold: a toy model for a cosmological singularity," hep-th/0202187.

[10] H. Liu, G. Moore and N. Seiberg, "Strings in a time-dependent orbifold," hepth/0204168.

[11] B. Craps, D. Kutasov and G. Rajesh, "String propagation in the presence of cosmological singularities," hep-th/0205101.

[12] C. Kounnas and D. Lust, "Cosmological string backgrounds from gauged WZW models", Phys. Lett. B289 (1992) 56, hep-th/9205046.

[13] L. Cornalba and M.S. Costa, "A new cosmological scenario in string theory", hepth/0203031.

[14] L. Cornalba, M.S. Costa and C. Kounnas, "A resolution of the cosmological singularity with orientifolds", hep-th/0205261.

[15] G.T. Horowitz and A.R. Steif, "Strings in strong gravitational backgrounds," Phys. Rev. D42 (1990) 1950.

[16] I learned this from various conversations at Stanford, and various Stanford ITP group meetings. The apparent culprits are: A. Adams, M. Fabinger, S. Giddings, S. Hellerman, M. Kleban, S. Kachru, N. Kaloper, J. McGreevy, S. Shenker, E. Silverstein and L. Susskind.

[17] O. Aharony, M. Fabinger, G.T. Horowitz and E. Silverstein, "Clean time dependent string backgrounds from bubble baths," hep-th/0204158.

[18] A.E. Lawrence and E.J. Martinec, "String field theory in curved spacetime and the resolution of spacelike singularities," Class. Quant. Grav. 13 (1996) 63; hep-th/9509149.

[19] G. Gibbons, Comm. Math. Phys. 45 (1976) 191. 
[20] J. Khoury, B.A. Ovrut, N. Seiberg, P.J. Steinhardt and N. Turok, "From big crunch to big bang," Phys. Rev. D65 (2002) 086007; hep-th/0108187.

[21] N. Seiberg, "From a big crunch to a big bang: is it possible?", hep-th/0201039.

[22] Y. Peleg and A. Steif, "Phase transition for gravitationally collapsing dust shells in $2+1$ dimensions," Phys. Rev. D51 (1995) R3992; gr-qc/9412023.

[23] M. Bañados, C. Teitelboim and J. Zanelli, "The black hole in three dimensional spacetime," Phys. Rev. Lett. 69 (1992) 1849, hep-th/9204099; M. Bañados, M. Henneaux, C. Teitelboim and J. Zanelli, "Geometry of the $2+1$ black hole," Phys. Rev. D48 (1993) 1506, gr-qc/9302012.

[24] L. Susskind, L. Thorlacius and J. Uglum, "The stretched horizon and black hole complementarity," Phys. Rev. D48 (1993) 3743, hep-th/9306069; L. Susskind, "String theory and the principles of black hole complementarity," Phys. Rev. Lett. 71 (1993) 2367, hep-th/9307068. 